\title{
Association between blood cadmium levels and the risk of osteopenia and osteoporosis in Korean post-menopausal women
}

\author{
Eun-San Kim ${ }^{1} \cdot$ Sangah $\operatorname{Shin}^{2} \cdot$ Yoon Jae Lee ${ }^{1} \cdot$ In-Hyuk Ha ${ }^{1}$ (D) \\ Received: 18 September 2020 / Accepted: 6 January 2021 / Published online: 2 February 2021 \\ (C) The Author(s) 2021
}

\begin{abstract}
Summary We aimed to investigate the association between cadmium levels and the risk of osteopenia and osteoporosis in Korean post-menopausal women. There was a significant positive association between cadmium levels and the risk of osteopenia and osteoporosis, but further studies for dose response are required.

Purpose Cadmium exposure can exert detrimental effects on bone health, particularly in post-menopausal women. However, previous studies have failed to report an association in Korean post-menopausal women. We aimed to investigate the association between cadmium levels and the risk of osteopenia and osteoporosis in Korean post-menopausal women.

Methods In total, 5432 participants from the 4th and 5th Korean National Health and Nutrition Examination Survey (KNHANES) were randomly sampled for measurements of heavy metal concentrations in the blood, bone mass density (BMD), and nutrient intake. We analyzed data for 1031 post-menopausal women $\geq 50$ years of age. Blood cadmium levels were categorized into quartiles, and a multinomial logistic regression model was used for analysis.

Results There was a significant positive association between cadmium levels and the risk of osteopenia and osteoporosis, but the odds ratio (OR) at the 4th level was lower than that at the 3rd level (OR and 95\% confidence interval (CI) for osteopenia: 2nd quartile: $1.24,0.88-1.74$; 3rd quartile: $3.22,2.24-4.64 ; 4$ th quartile: $1.27,0.87-1.85 ; P$ for trend $<0.001$; OR and $95 \%$ CI for osteoporosis: 2nd quartile: $1.54,1.05-2.25$; 3rd quartile: $3.63,2.31-5.69 ; 4$ th quartile: $1.70,1.03-2.81 ; P$ for trend $<0.001)$. This trend was consistent in the sensitivity analysis.

Conclusion Our findings suggest that there is an association between blood cadmium levels and the risk of osteopenia and osteoporosis in Korean post-menopausal women. However, further prospective studies are required to determine whether there is a dose-response relationship and address potential selection bias, especially in patients with femoral neck osteoporosis.
\end{abstract}

Keywords Cadmium · Osteoporosis · Osteopenia $\cdot$ Post-menopausal women

\section{Introduction}

Cadmium exposure is a major risk factor for osteoporosis. Cadmium can accumulate in the body via occupation-related exposure, smoking, diet, and other sources [1]. Cadmium disturbs the mineralization of bone by damaging the renal tubular system, interfering with parathyroid hormone (PTH) and

In-Hyuk Ha

hanihata@gmail.com

1 Jaseng Spine and Joint Research Institute, Jaseng Medical Foundation, 3F, 538 Gangnam-daero, Gangnam-gu, Seoul 06110, Republic of Korea

2 Department of Food and Nutrition, Chung-Ang University, Gyeonggi-do 17546, Republic of Korea vitamin D metabolism, reducing absorption of calcium from the intestine, and directly damaging the bone $[2,3]$. The risk of cadmium exposure has been identified in various populations [4-6], including post-menopausal women [7, 8], who are particularly vulnerable to osteoporosis [9].

However, previous studies have failed to identify a positive association between cadmium exposure and osteoporosis in Korean post-menopausal women [10]. We attempted to supplement this work using various methods: More samples were included to reduce random bias, multiple imputations were used for missing data to reduce selection bias, the weights were adjusted given the complex survey design [11], a multinomial model was used for precise estimation of associations [6], and the model was constructed in consideration of exposure, confounders, and outcomes [12]. Therefore, in the present study, we aimed to investigate the association between 
cadmium exposure and the risk of osteopenia and osteoporosis in Korean post-menopausal women to address the remaining gaps in knowledge.

\section{Methods}

\section{Participants}

The present study utilized samples from the 4th and 5th Korean National Health and Nutrition Examination Survey (KNHANES). The original data were obtained from July 2008 to May 2011. Heavy metal levels, bone mass density (BMD), general health, and nutrition were assessed during this period. The KNHANES is a nationwide cross-sectional survey conducted each year by the Korea Centers for Disease Control and Prevention. The goal of the KNHANES is to assess and monitor health and nutritional status in the Republic of Korea. The target population consists of all noninstitutionalized citizens residing in Korea. The KNHANES utilizes a complex survey design and is constructed via twostage stratified cluster sampling. The KNHANES represents the target population using sampling weights. Additional details have been described in a previous study [13].

The population of the present study consisted of postmenopausal women $\geq 50$ years of age. Among 37,753 participants in the 2008-2011 KNHANES, a total of 5432 participants were randomly sampled for measurements of heavy metal concentrations in the blood, BMD, and nutrient intake. Male participants $(n=2569)$, those under age $50(n=1766)$, and nonpost-menopausal women $(n=66)$ were excluded from the analysis. Multiple imputations were then used for missing covariates in the remaining 1031 participants (Fig. 1). Other exclusion criteria (e.g., early menopause, cancer) were not adopted, as these variables are effect modifiers or mediators [14] rather than confounders in the present model $[15,16]$ (Online Resource 1).

\section{Blood cadmium levels}

Blood samples were obtained from participants for the measurement of blood cadmium levels. Samples were collected in vacuum tubes (vacutainer), mixed with anticoagulants, and stored in a refrigerator. They were analyzed within $24 \mathrm{~h}$ in the diagnostic laboratory of NeoDin Medical Institute in Seoul, South Korea. Blood levels of cadmium were measured via graphite furnace atomic absorption spectrometry (GFAAS) using a Perkin Elmer AAnalyst 600 system (PerkinElmer, Turku, Finland). In the internal validation step, inter-assay coefficients of variation (CVs) continued to be within an acceptable range $(\leq 10 \%)$. External validation was performed by the Korea Occupational Safety and Health Agency and German External Quality Assessment Scheme (G-EQUAS). The benchmark dose of blood cadmium has yet to be determined for the Korean post-menopausal population. Therefore, blood cadmium levels were categorized into quartiles following previous study [10].

\section{BMD, osteopenia, and osteoporosis}

BMD was measured for the total hip, femoral neck, and lumbar spine (L1-L4) using dual-energy X-ray absorptiometry (DXA, DISCOVERY-W fan-beam densitometer; Hologic Inc., Bedford, MA, USA). For precision assessment, radiologic technologists were trained to make the CVs of randomly double-checked participants $\leq 1.8 \%$ for the total hip, $\leq 2.5 \%$ for the femoral neck, and $\leq 1.9 \%$ for the lumbar spine.
Fig. 1 Flowchart of participant selection

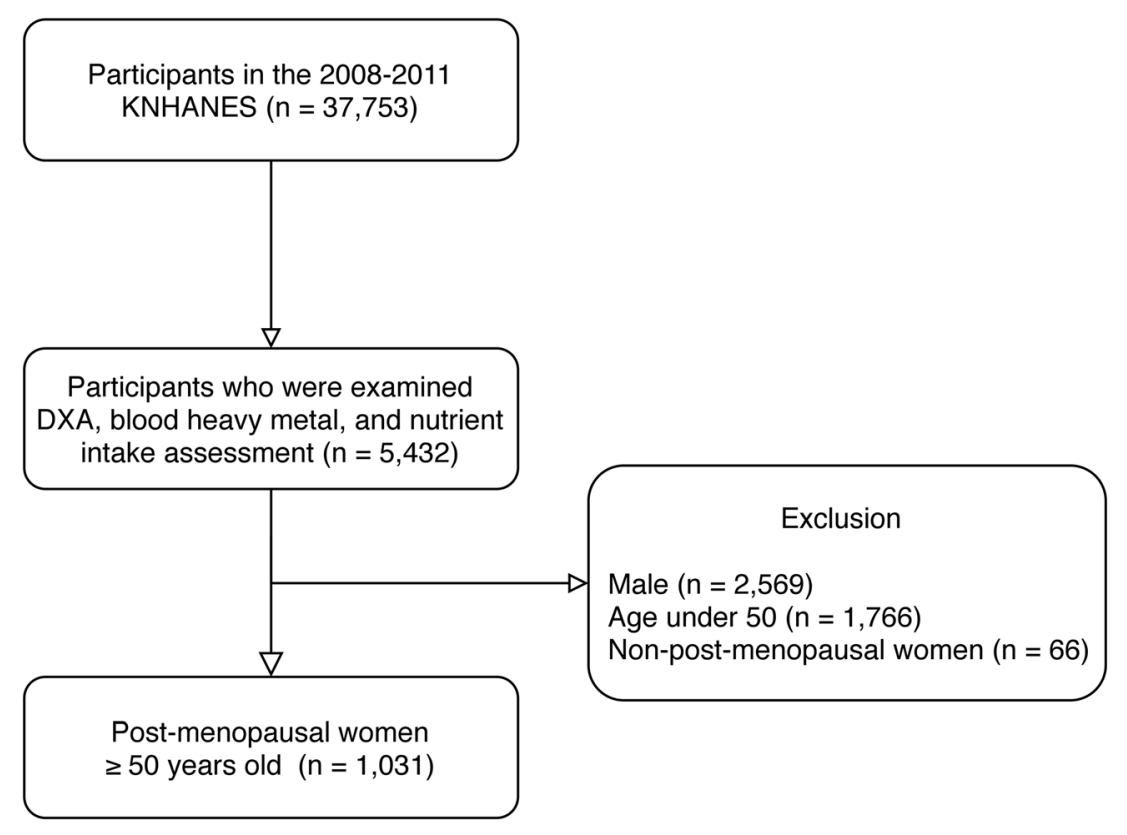


Osteoporosis and osteopenia were defined according to $\mathrm{T}$ scores. The T-score is the standard deviation from the average peak BMD for sex-matched and race-matched (Japanese) populations [17]. Osteoporosis was defined as a T-score of -2.5 or less at any site, while osteopenia was defined as a Tscore less than -1.0 and greater than -2.5 .

\section{Confounders}

To minimize confounding bias, we selected confounders based on the disjunctive cause criterion. In this criterion, variables that are cause of exposure, outcome, or both are controlled. If exposure is the cause of a variable, it is regarded as a mediator or collider, rather than a confounder. Adjusting these variables would lead to bias [18]. A detailed explanation of confounder selection is presented in the form of a directed acyclic graph (DAG) (Online Resource 1).

Age, education level, household income (quartile), occupation, and residence were included as confounders reflecting socioeconomic status $[1,19]$. To minimize residual confounding, age was adjusted as both a continuous and categorical variable. The year in which participants were surveyed was also adjusted [20].

Body mass index (BMI) was calculated based on measured height and weight [21]. Lifestyle factors such as current alcohol consumption, smoking exposure, and physical activity were also included [1, 19, 22]. When defining smoking exposure, we considered that women smokers tend to under-report their smoking status by more than $50 \%$, as identified by a previous KNHANES [23]. Therefore, we defined smoking exposure as $50 \mathrm{ng} / \mathrm{mL}$ or more urinary cotinine, based on the results of previous studies [24, 25]. Gas chromatography-mass spectrometry (GC-MS) with a Clarus 600/600 T system (Perkin Elmer, Waltham, MA, USA) was used to measure urinary cotinine. Other information was obtained using questionnaires. Alcohol consumption was categorized into "less than once per month," "greater than once per month but less than twice per week," and "greater than twice per week" [26]. Physical activity was categorized as vigorous (3 days/week, $\geq 20 \mathrm{~min} / \mathrm{session}$ ), moderate (5 days/week, $\geq 30 \mathrm{~min} / \mathrm{session}$ ), or daily walking (5 days/week, $\geq 30 \mathrm{~min} / \mathrm{session}$ ).

Vitamin D levels were measured based on serum 25hydroxyvitamin $\mathrm{D}(25(\mathrm{OH}) \mathrm{D} 3)$ concentration using a radioimmunoassay (1470 WIZARD gamma-Counter, PerkinElmer, Turku, Finland) [27]. Food consumptions were recorded based on the 24-h dietary recall method, following which nutrient intake was calculated. Fish and seaweed consumption [28] as well as energy, protein, calcium, phosphate, potassium, and retinol intake [29] were included in the model. Intake of certain foods was classified as less than once a week, once a week, or more than once a week [30]. Nutrient intake was adjusted as a continuous variable. The previous use of hormone therapy or oral contraceptive ( $>1$ month of use) was obtained using a questionnaire. The use of hormone therapy was categorized into "never," "less than 2 years," and "2 years or more" [31, 32].

\section{Statistical analysis}

Among the study population ( $n=1031), 122$ participants $(11.8 \%)$ had at least one missing covariate or outcome value. Multiple imputations were used to impute item non-response. Assuming missing at random (MAR), all variables in the model were included in the multiple imputations. For continuous variables, we used predictive mean matching due to their nonnormal distribution. For categorical variables, we used different types of logistic regression for categorical variables based on the variable type (i.e., binary, multinomial, or ordinal). Imputation was performed using the Markov chain Monte Carlo (MCMC) method, and 20 imputed sets were created.

The design and sampling weights of all participants in the KNHANES were included in the analysis to account for the complex survey design. Multinomial logistic regression was used to analyze the associations between cadmium and the risk of osteopenia and osteoporosis. Blood cadmium levels were divided into quartiles, as previously described [10]. In the multinomial model, we used replicate weights with the bootstrap method to estimate $95 \%$ confidence intervals (CIs). Bootstrapping was performed 250 times with five imputed sets each. Regression methods were used to examine linear $P$ trends in blood cadmium levels.

\section{Exploratory and sensitivity analyses}

For exploratory purposes, we investigated the association of cadmium with osteopenia and osteoporosis by subregion. Osteopenia and osteoporosis in the total hip, femoral neck, and lumbar spine were defined according to T-scores. The multinomial model was respectively used in each subregion except for osteoporosis in total hip due to the low number of cases.

Sensitivity analyses were performed based on three different outcome definitions. (1) Some patients may undergo treatment following the development of osteoporosis, leading to an increase in T-score. If the proportion of treated patients varies based on cadmium levels, using only current $\mathrm{T}$-scores to define osteoporosis can lead to biased estimates. Therefore, patients with T-scores greater than -2.5 answering "yes" to "Have you ever been diagnosed with osteoporosis by a doctor?" and "Are you currently under treatment for osteoporosis?" were classified into the osteoporosis group. (2) The reference for calculating $\mathrm{T}$-scores was derived from a Japanese population [17]; however, reference values may differ for Korean and Japanese populations [33]. Therefore, osteopenia and osteoporosis were defined according to T-scores that had been re-calculated using Korean reference values. (3) T-scores 
based on Korean reference values and current treatment information were both used to define osteoporosis and osteopenia.

To examine the robustness of the results, additional sensitivity analysis was performed. (1) Blood cadmium level was categorized into quintile (five sections). (2) Outcome was defined as T-score -1.8 or less and -2.0 or less. For each outcome, logistic regression was used. (3) BMD and T-score at each site were analyzed. Moreover, the lowest BMD and Tscore among sites were analyzed.

\section{Ethical approval}

The study protocol was approved by the Institutional Review Board of Jaseng Hospital of Korean Medicine (JASENG 2020-08-019), who waived the requirement for informed consent due to the nature of the study.

\section{Results}

The general characteristics of the participants are presented in Table 1. Mean blood cadmium levels were $0.81 \pm 0.02$ (mean $\pm \mathrm{SE})$ in the 1 st quartile and $2.43 \pm 0.05($ mean $\pm \mathrm{SE})$ in the 4 th quartile. Level of education, household income, phosphate intake, and retinol intake decreased as cadmium levels increased. Moreover, the prevalence of smoking exposure was higher in the 3rd and 4th quartiles than in the 1st and 2nd quartiles.

The prevalence rates and odds ratios (ORs) for osteoporosis and osteopenia are presented according to cadmium levels in Table 2. Higher cadmium levels were significantly associated with both osteopenia and osteoporosis, except the OR for osteopenia in the 4th quartile. The ORs for osteopenia and osteoporosis decreased at the 4th quartile but the $P$ trend indicated significant positive association. ORs were generally higher for osteoporosis than for osteopenia (OR and 95\% CI for osteopenia, 2nd quartile: 1.24, 0.88-1.74; 3rd quartile: $3.22,2.24-4.64$; 4 th quartile: $1.27,0.87-1.85 ; P$ for trend $<0.001$; for osteoporosis, 2 nd quartile: $1.54,1.05-2.25$; $3 \mathrm{rd}$ quartile: $3.63,2.31-5.69 ;$ th quartile: $1.70,1.03-2.81 ; P$ for trend $<0.001$ ).

The trend of association was generally consistent in the exploratory and sensitivity analyses. However, when the exploratory analysis was performed by subregion (Fig. 2), the OR for the femoral neck in the 4th quartile was significantly lower than 1 for osteopenia. Moreover, the $P$ trend indicated a significant negative association for osteoporosis in the femoral neck (OR in the 4th quartile and 95\% CI for osteopenia of femoral neck: $0.79,0.64-0.99 ; P$ for trend of osteoporosis in femoral neck: 0.009).

In the first sensitivity analysis, which considered current treatment in the outcome definition, the ORs for osteoporosis slightly increased in the 3rd and 4th quartiles (OR and 95\% CI for osteoporosis, 3rd quartile: 3.90, 2.46-6.16; 4th quartile: $1.85,1.14-2.99)$. However, when T-scores were calculated using Korean reference values, the ORs generally decreased, and only the ORs for osteoporosis in the 3rd quartile remained significant (OR and 95\% CI for osteoporosis, 3rd quartile: $1.80,1.16-2.81)$. When current treatment and Korean reference values were both considered, the ORs for osteoporosis increased and 4th quartile became significant (OR and 95\% CI for osteoporosis: 3rd quartile: 1.92, 1.39-2.67, 4th quartile: $1.72,1.10-2.69)$. The $P$ trend indicated significant positive association in all models (Table 3 ).

In the analysis performed with the quintile, the OR in the 3 rd and 4th quintile was significant for osteopenia and osteoporosis, but the OR decreased in the 5th quintile and became non-significant (OR and 95\% CI for osteopenia, 3rd quintile: $2.45,1.49-4.01 ; 4$ th quintile: $1.69,1.02-2.82 ; 5$ th quintile: $1.20,0.75-1.93$; $P$ for trend $<0.001$; for osteoporosis, $3 \mathrm{rd}$ quartile: $2.42,1.31-4.49 ; 4$ th quartile: $2.83,1.48-5.40 ; 5$ th quartile: $1.66,0.86-3.19 ; P$ for trend $<0.001$ ) (Online Resource 2). An analysis with T-score -2.0 or less showed significant $\mathrm{OR}$ in the 4th quartile and the trend showed dose response (OR and $95 \% \mathrm{CI}$ for $\mathrm{T}$-score -2.0 or less, 4th quartile: 1.76, 1.02-3.05; $P$ for trend: 0.027) (Online Resource 3). When BMD and T-score were analyzed as outcomes, the 3rd quartile was significant only for lowest $\mathrm{T}$-score among sites (estimates and $95 \% \mathrm{CI}$ for lowest T-score, 3rd quartile: -0.17 , -0.34 to -0.01 ) (Online Resource 4).

\section{Discussion}

Our findings indicated that the risks of osteopenia and osteoporosis increased as blood cadmium levels increased. However, the OR at the highest (4th quartile) blood cadmium level was lower than that at the 3rd quartile, despite a significant $P$ trend, making the dose-response relationship somewhat unclear. This trend was consistent with that observed in the sensitivity analysis performed using Korean reference values for T-scores. Thus, although we concluded that blood cadmium levels affect the risk of osteoporosis and osteopenia in post-menopausal women, our findings should be interpreted with caution.

There are some explanations for the absence of a doseresponse relationship at the highest cadmium levels. First, when current treatment information was considered in the definition of osteoporosis, the OR for osteoporosis at the $3 \mathrm{rd}$ and 4th cadmium level increased, suggesting that patients with osteoporosis exhibiting higher cadmium levels tended to utilize medical care more, potentially decreasing T-scores. Such findings further indicate that patients with osteoporosis with higher cadmium levels may be relatively aware of their risk, although this hypothesis is difficult to verify using the current 
Table 1 Basic characteristics of the study population

Blood cadmium levels

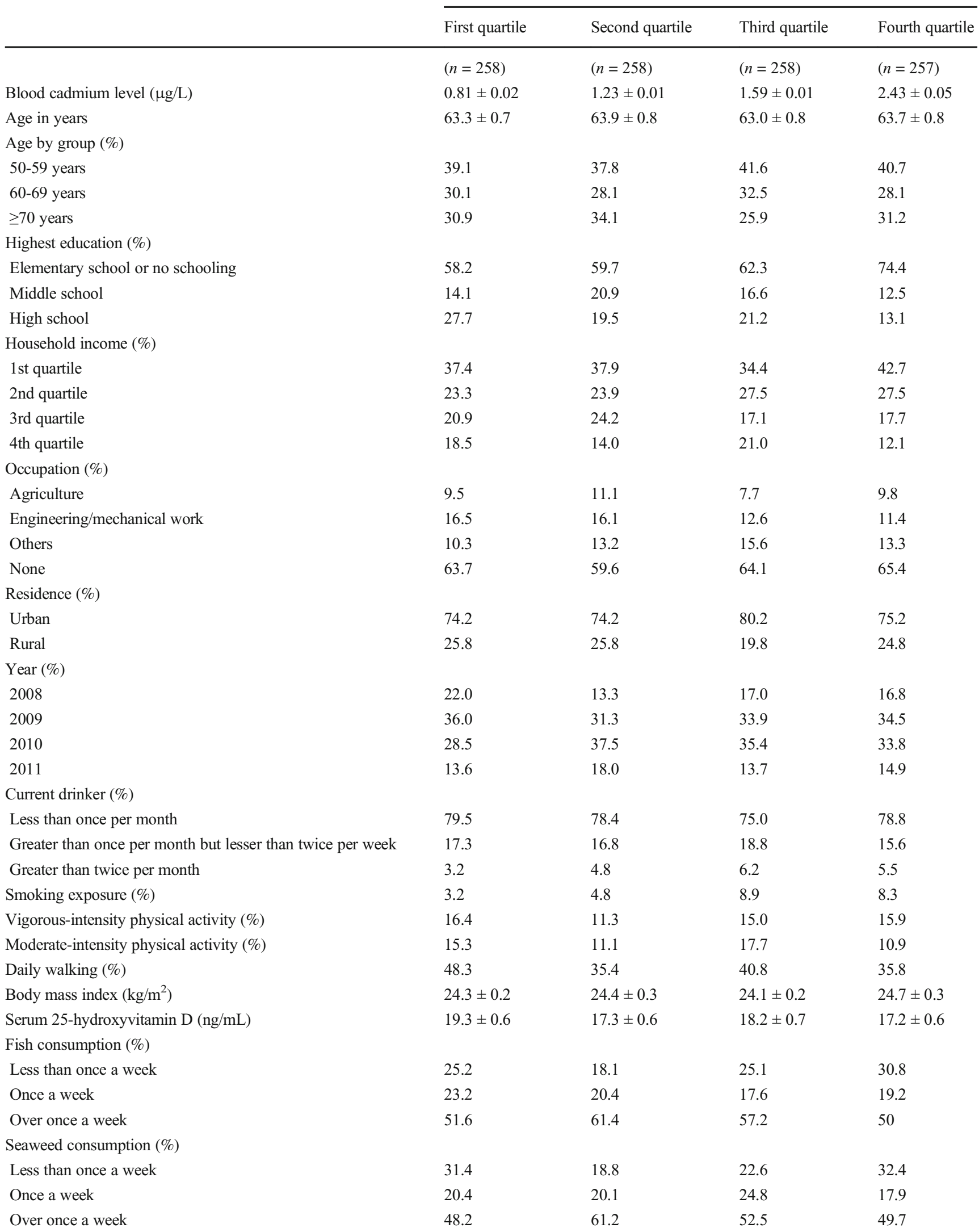


Table 1 (continued)

\begin{tabular}{|c|c|c|c|c|}
\hline & \multicolumn{4}{|c|}{ Blood cadmium levels } \\
\hline & First quartile & Second quartile & Third quartile & Fourth quartile \\
\hline Energy intake per day (Kcal) & $1571.0 \pm 42.1$ & $1549.0 \pm 51.4$ & $1519.9 \pm 44.2$ & $1551.2 \pm 47.7$ \\
\hline Protein intake per day (g) & $53.8 \pm 2.0$ & $50.9 \pm 2.1$ & $51.7 \pm 2.2$ & $49.2 \pm 1.9$ \\
\hline Calcium intake per day (mg) & $434.9 \pm 22.1$ & $383.4 \pm 18.6$ & $455.0 \pm 32.6$ & $380.9 \pm 20.1$ \\
\hline Phosphate intake per day (mg) & $983.0 \pm 31.5$ & $934.2 \pm 33.3$ & $932.8 \pm 37.2$ & $916.7 \pm 32.5$ \\
\hline Potassium intake per day (mg) & $2753.7 \pm 106.8$ & $2535.1 \pm 107.7$ & $2486.1 \pm 98.9$ & $2620.8 \pm 128.4$ \\
\hline Retinol intake per day $(\mu g)$ & $94.9 \pm 23.7$ & $51.0 \pm 5.4$ & $53.3 \pm 6.5$ & $44.2 \pm 7.1$ \\
\hline Oral contraceptive use (\%) & 21.7 & 23.6 & 29.8 & 26.3 \\
\hline \multicolumn{5}{|l|}{ Hormone replacement therapy $(\%)$} \\
\hline Never & 82.2 & 73.8 & 84.9 & 90.1 \\
\hline Less than 2 years & 8.5 & 12.8 & 9.1 & 5.8 \\
\hline 2 years or more & 9.4 & 13.4 & 6.0 & 4.1 \\
\hline
\end{tabular}

Cadmium exposure was divided into quartiles. The cut-off points for quartiles were $1.04 \mu \mathrm{g} / \mathrm{L}, 1.40 \mu \mathrm{g} / \mathrm{L}$, and $1.82 \mu \mathrm{g} / \mathrm{L}(1.01 \mu \mathrm{g} / \mathrm{L}, 1.36 \mu \mathrm{g} / \mathrm{L}, 1.78$ $\mu \mathrm{g} / \mathrm{L}$ in weighted value for complex survey design). The basic characteristics are presented as the mean \pm standard error of the mean for continuous variables and as percentages for categorical variables

data. Furthermore, the OR at the highest (4th) level was still lower than that at the 3 rd level.

Second, our results may have also been influenced by selection bias. The target population of the KNHANES only includes non-institutionalized citizens, meaning that patients with severe comorbidities may have been excluded from the survey. For example, cadmium is a known risk factor for stroke [34] and cancer [16], both of which increase the risk of osteoporosis $[35,36]$. Patients experiencing long-term admission due to these diseases may have been excluded, which in turn may have resulted in selection bias at the highest cadmium level. In our subregion analysis, negative associations were observed for the femoral neck, indicating that selection bias may have occurred with regard to patients with femoral neck osteoporosis.

There were various results with the additional sensitivity analysis. According to our quintile analysis, there was a dose- response relationship until the 4th quintile, although it disappeared in the 5th quintile. Assuming that selection bias caused the dose-response relationship to disappear, this supports the hypothesis that it may have occurred in groups with high cadmium exposure. In addition, a weak dose-response relationship was observed in analyses performed using a T-score less than or equal to -2.0 (defined by mitigating the criteria for abnormal status). If selection bias occurred among individuals with osteoporosis in the higher cadmium exposure, it is possible that this approach has alleviated this problem to some extent.

Blood cadmium levels were relatively lower among our patients than among those in previous studies $[5,37]$. This discrepancy may be due to the inclusion of a nationally representative population in our study, in contrast to previous studies, which have focused on populations living in polluted regions. However, the risk of osteoporosis was noted even at

Table 2 Association between blood cadmium levels and the risk of osteopenia and osteoporosis

\begin{tabular}{|c|c|c|c|c|c|c|}
\hline \multirow[b]{2}{*}{ BCd level } & \multicolumn{3}{|l|}{ Osteopenia } & \multicolumn{3}{|l|}{ Osteoporosis } \\
\hline & Prevalence & Unadjusted & Adjusted & Prevalence & Unadjusted & Adjusted \\
\hline First quartile & $50.55(42.42-58.67)$ & Ref & Ref & $33.90(26.21-41.58)$ & Ref & Ref \\
\hline Second quartile & $47.64(39.34-55.94)$ & $1.06(0.85-1.32)$ & $1.24(0.88-1.74)$ & $38.33(29.86-46.80)$ & $1.26(0.98-1.63)$ & $1.54(1.05-2.25)$ \\
\hline Third quartile & $55.94(47.60-64.28)$ & $2.16(1.64-2.85)$ & $3.22(2.24-4.64)$ & $36.06(27.90-44.22)$ & $2.07(1.58-2.73)$ & $3.63(2.31-5.69)$ \\
\hline Fourth quartile & $45.25(36.76-53.74)$ & $1.05(0.82-1.34)$ & $1.27(0.87-1.85)$ & $41.47(32.41-50.53)$ & $1.42(1.10-1.84)$ & $1.70(1.03-2.81)$ \\
\hline$P$ for trend & & & $<0.001$ & & & $<0.001$ \\
\hline
\end{tabular}

The association between blood cadmium levels and the risk of osteopenia and osteoporosis was analyzed using a multinomial logistic regression model. Osteopenia and osteoporosis were defined based on T-scores. The prevalence rates and odds ratios (ORs) are presented with $95 \%$ confidence intervals for each quartile. BCd: blood cadmium 
Fig. 2 Secondary analysis of associations between blood cadmium levels and the risk of osteopenia and osteoporosis by subregion. We performed a secondary analysis to investigate the association between cadmium levels and the risk of osteopenia and osteoporosis by subregion. Osteopenia and osteoporosis in the total hip, femoral neck, and lumbar spine were defined according to $\mathrm{T}$-scores (osteoporosis: -2.5 or less; osteopenia: less than -1.0 and greater than-2.5). A multinomial logistic regression model was used to assess osteopenia and osteoporosis in each subregion. The prevalence rates and odds ratios (ORs) are presented with 95\% confidence intervals (CIs) for each quartile. * The $P$ trend for osteoporosis of the femoral neck was significant, but the association was negative. $\mathrm{BCd}$ : blood cadmium a

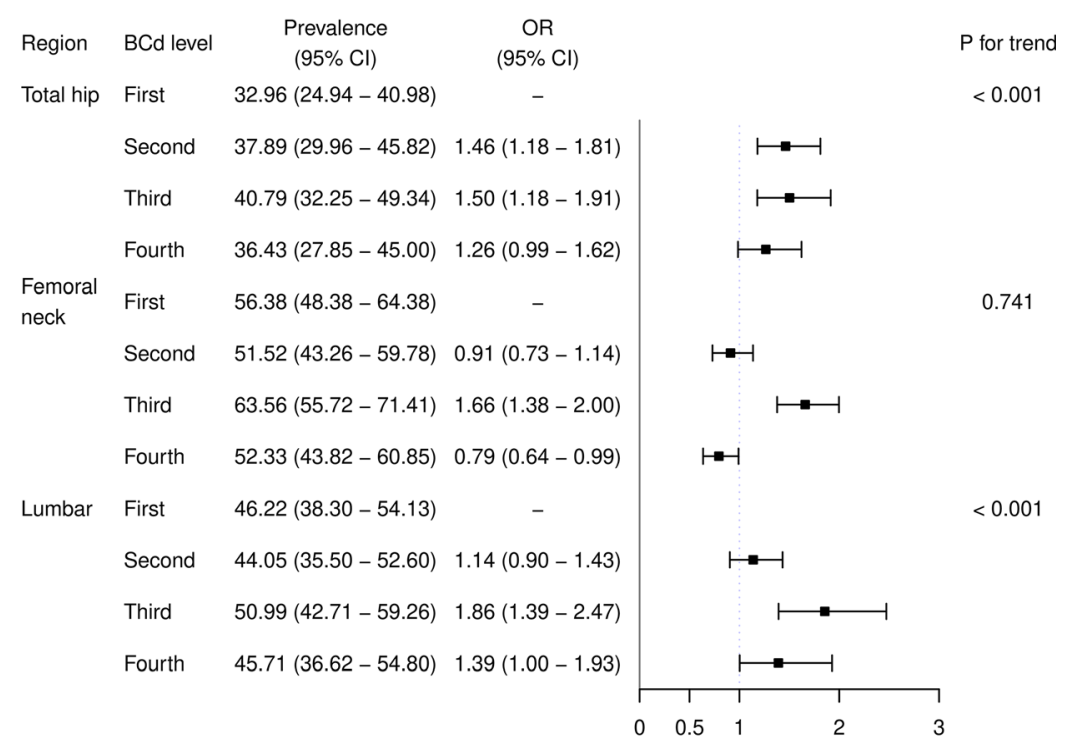

b

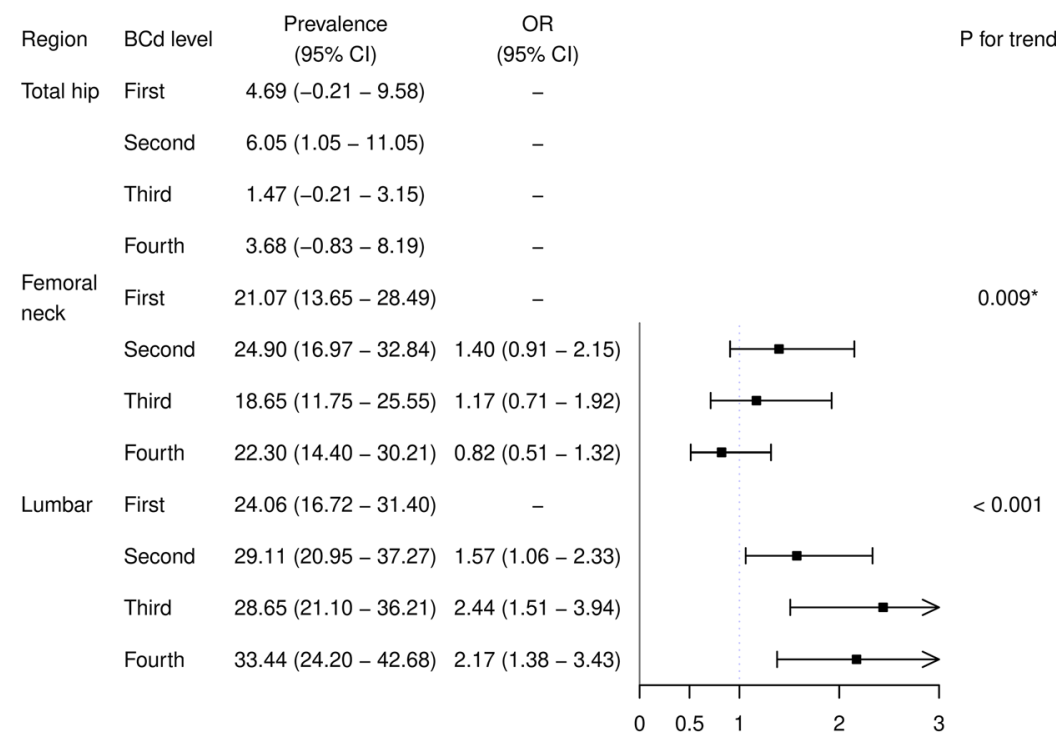

[41]. Therefore, it is difficult to determine the appropriate mediators for the model.

The strength of our study is that we demonstrated the possibility of an association between cadmium exposure and bone health in Korean post-menopausal women by complementing previous study using various methods. In addition to increasing sample size, utilizing multiple imputations and multinomial models, and adjusting weights given the complex survey design, we considered causal mechanisms in constructing our model. The disjunctive cause criterion is recommended for confounder selection when one cannot know all causal mechanisms related to exposures or outcomes [18]. Bias can occur if the cause of a variable is cadmium exposure (e.g., cancer [16], hypertension [42], or kidney function [2, 39]) or osteoporosis (e.g., fracture or osteoporosis medication) and the variable is controlled via exclusion or adjustment. Therefore, we 
Table 3 Sensitivity analysis for different outcome definitions of osteopenia and osteoporosis

\begin{tabular}{|c|c|c|c|c|c|c|}
\hline \multirow[b]{2}{*}{ BCd level } & \multicolumn{3}{|l|}{ Osteopenia } & \multicolumn{3}{|l|}{ Osteoporosis } \\
\hline & Prevalence & Unadjusted & Adjusted & Prevalence & Unadjusted & Adjusted \\
\hline \multicolumn{7}{|l|}{ Model 1} \\
\hline First quartile & $46.82(38.75-54.89)$ & Ref & Ref & $37.62(29.87-45.38)$ & Ref & Ref \\
\hline Second quartile & $43.28(35.10-51.46)$ & $0.99(0.77-1.28)$ & $1.24(0.90-1.69)$ & $42.69(34.23-51.15)$ & $1.25(0.99-1.58)$ & $1.61(1.10-2.37)$ \\
\hline Third quartile & $49.61(41.25-57.97)$ & $2.10(1.54-2.86)$ & $3.14(2.14-4.60)$ & $42.39(33.97-50.81)$ & $2.23(1.69-2.96)$ & $3.90(2.46-6.16)$ \\
\hline Fourth quartile & $41.56(33.48-49.64)$ & $1.08(0.81-1.45)$ & $1.34(0.88-2.03)$ & $45.83(36.95-54.71)$ & $1.47(1.12-1.94)$ & 1.85 (1.14-2.99) \\
\hline$P$ for trend & & & $<0.001$ & & & $<0.001$ \\
\hline \multicolumn{7}{|l|}{ Model 2} \\
\hline First quartile & $48.69(40.39-56.99)$ & Ref & Ref & $29.75(22.15-37.34)$ & Ref & Ref \\
\hline Second quartile & $51.85(43.10-60.59)$ & $1.11(0.91-1.36)$ & $1.13(0.90-1.41)$ & $27.46(19.32-35.59)$ & $0.95(0.74-1.21)$ & $0.98(0.67-1.43)$ \\
\hline Third quartile & $53.65(45.25-62.06)$ & $1.45(1.21-1.75)$ & $1.66(1.30-2.11)$ & $29.95(22.06-37.85)$ & $1.29(1.04-1.59)$ & $1.80(1.16-2.81)$ \\
\hline Fourth quartile & $47.22(38.68-55.76)$ & $1.16(0.96-1.40)$ & $1.25(0.92-1.68)$ & $34.78(25.58-43.97)$ & $1.38(1.06-1.78)$ & $1.50(0.95-2.38)$ \\
\hline$P$ for trend & & & $<0.001$ & & & $<0.001$ \\
\hline \multicolumn{7}{|l|}{ Model 3} \\
\hline First quartile & $44.94(36.71-53.18)$ & Ref & Ref & 33.49 (25.79-41.19) & Ref & Ref \\
\hline Second quartile & $44.91(36.16-53.66)$ & $1.02(0.85-1.23)$ & $1.06(0.83-1.36)$ & $34.40(25.88-42.91)$ & $1.05(0.85-1.30)$ & $1.13(0.79-1.60)$ \\
\hline Third quartile & $48.22(39.83-56.61)$ & $1.48(1.18-1.84)$ & $1.69(1.30-2.19)$ & $36.02(27.63-44.41)$ & $1.48(1.19-1.83)$ & $1.92(1.39-2.67)$ \\
\hline Fourth quartile & $41.22(33.11-49.33)$ & $1.14(0.92-1.42)$ & $1.20(0.86-1.65)$ & $41.45(32.33-50.57)$ & $1.56(1.20-2.02)$ & $1.72(1.10-2.69)$ \\
\hline$P$ for trend & & & $<0.001$ & & & $<0.001$ \\
\hline
\end{tabular}

Model 1: T-score criteria (osteoporosis: -2.5 or less; osteopenia: less than -1.0 and greater than -2.5 ) and current treatment information (patients with Tscores greater than -2.5 but currently undergoing osteoporosis treatment were classified as having osteoporosis). Model 2: T-score criteria calculated using reference values for the Korean population. Model 3: T-score criteria calculated using Korean reference values and current treatment information. The prevalence rates and odds ratios (ORs) are presented with 95\% confidence intervals for each quartile. BCd: blood cadmium

only considered confounders that were the cause of exposure, outcomes, or both.

There are additional limitations in our study. First, blood cadmium level was used as index of cadmium exposure. However, blood cadmium is predominantly influenced by recent cadmium exposure. Urinary cadmium levels may therefore be more appropriate for investigating osteoporosis given their long-term stability [43]. However, the KNHANES did not assess urinary cadmium levels. Furthermore, blood and urinary cadmium levels are highly correlated [44], and several previous studies have utilized blood cadmium levels to investigate changes in BMD [5, 37, 45]. Moreover, cadmium excretion can occur when patients experience tubular proteinuria, which may make measurement of blood cadmium levels more appropriate [46]. Second, there may have been issues with confounder selection due to the use of cross-sectional data. For example, serum 25(OH)D3 level was used as an index of vitamin D deficiency. If the conversion of $25(\mathrm{OH}) \mathrm{D} 3$ to calcitriol is disturbed by cadmium, serum levels of 25(OH)D3 may be affected [2]. In this case, cadmium acts as a mediator, rather than a confounder [14]. Furthermore, it is possible that patients underwent hormone replacement therapy due to cadmium-induced osteoporosis caused by cadmium. Controlling for this variable would thus induce collider bias
[18]. Further prospective studies are required to provide nonbiased estimates.

\section{Conclusion}

Our findings demonstrate that there may be a positive association between cadmium levels and the risk of osteopenia and osteoporosis in Korean menopausal women. However, further prospective studies are required to explain the absence of a dose-response relationship and address potential selection bias.

Supplementary Information The online version contains supplementary material available at https://doi.org/10.1007/s11657-021-00887-9.

Author contribution E.S.K: conceptualization, design, writing (original draft), data curation, methodology, and formal analysis; S.A.S and Y.J.L: writing (review and editing); I.H.H: supervision and writing (review and editing).

Availability of data and material KNHANES data were provided by the Korea Centers for Disease Control and Prevention. Researchers can freely access KNHANES data using the following link: https://knhanes.cdc.go. $\mathrm{kr} / \mathrm{knhanes} /$ main.do. 
Code availability R studio (version 1.1.463) was used for analysis.

\section{Declarations}

Ethics approval The study protocol was approved by the Institutional Review Board of Jaseng Hospital of Korean Medicine (JASENG 202008-019).

Consent to participate The Institutional Review Board of Jaseng Hospital of Korean Medicine waived the requirement for informed consent due to the nature of the study.

\section{Consent for publication Not applicable.}

\section{Conflicts of interest None.}

Open Access This article is licensed under a Creative Commons Attribution 4.0 International License, which permits use, sharing, adaptation, distribution and reproduction in any medium or format, as long as you give appropriate credit to the original author(s) and the source, provide a link to the Creative Commons licence, and indicate if changes were made. The images or other third party material in this article are included in the article's Creative Commons licence, unless indicated otherwise in a credit line to the material. If material is not included in the article's Creative Commons licence and your intended use is not permitted by statutory regulation or exceeds the permitted use, you will need to obtain permission directly from the copyright holder. To view a copy of this licence, visit http://creativecommons.org/licenses/by/4.0/.

\section{References}

1. Järup L, Berglund M, Elinder CG, Nordberg G, Vanter M (1998) Health effects of cadmium exposure-a review of the literature and a risk estimate. Scand J Work Environ Health:1-51

2. Kazantzis G (2004) Cadmium, osteoporosis and calcium metabolism. Biometals 17:493-498

3. Kjellström T (1992) Mechanism and epidemiology of bone effects of cadmium. IARC Sci Publ:301-310

4. Alfvén T, Elinder CG, Carlsson MD, Grubb A, Hellström L, Persson B, Pettersson C, Spång G, Schütz A, Järup L (2000) Low-level cadmium exposure and osteoporosis. J Bone Miner Res 15:1579-1586

5. Chen X, Wang K, Wang Z, Gan C, He P, Liang Y, Jin T, Zhu G (2014) Effects of lead and cadmium co-exposure on bone mineral density in a Chinese population. Bone 63:76-80

6. Wu Q, Magnus J, Hentz J (2010) Urinary cadmium, osteopenia, and osteoporosis in the US population. Osteoporos Int 21:14491454

7. Åkesson A, Bjellerup P, Lundh T, Lidfeldt J, Nerbrand C, Samsioe G, Skerfving S, Vahter M (2006) Cadmium-induced effects on bone in a population-based study of women. Environ Health Perspect 114:830-834

8. Gallagher CM, Kovach JS, Meliker JR (2008) Urinary cadmium and osteoporosis in US women $\geq 50$ years of age: NHANES 19881994 and 1999-2004. Environ Health Perspect 116:1338-1343

9. Tella SH, Gallagher JC (2014) Prevention and treatment of postmenopausal osteoporosis. J Steroid Biochem Mol Biol 142:155170

10. Cho GJ, Park HT, Shin JH, Hur JY, Kim SH, Lee KW, Kim T (2012) The relationship between blood mercury level and osteoporosis in postmenopausal women. Menopause 19:576-581
11. Kim Y, Park S, Kim N-S, Lee B-K (2013) Inappropriate survey design analysis of the Korean National Health and Nutrition Examination Survey may produce biased results. J Prev Med Public Health 46:96-104

12. Brewer LE, Wright JM, Rice G, Neas L, Teuschler L (2017) Causal inference in cumulative risk assessment: the roles of directed acyclic graphs. Environ Int 102:30-41

13. Kweon S, Kim Y, M-j J, Kim Y, Kim K, Choi S, Chun C, Khang YH, Oh K (2014) Data resource profile: the Korea national health and nutrition examination survey (KNHANES). Int J Epidemiol 43:69 77

14. Richiardi L, Bellocco R, Zugna D (2013) Mediation analysis in epidemiology: methods, interpretation and bias. Int J Epidemiol 42:1511-1519

15. Chen X, Zhu G, Jin T (2018) Effects of cadmium exposure on age of menarche and menopause. Toxics 6:6

16. Hartwig A (2013) Cadmium and cancer. From Toxicity to Essentiality. Springer, Cadmium, pp 491-507

17. Orimo H, Sugioka Y, Fukunaga M, Muto Y, Hotokebuchi T, Gorai I, Nakamura T, Kushida K, Tanaka H, Ikai T (1998) Diagnostic criteria of primary osteoporosis. J Bone Miner Metab 16:139-150

18. VanderWeele TJ (2019) Principles of confounder selection. Eur J Epidemiol 34:211-219

19. Tyrrell J, Melzer D, Henley W, Galloway TS, Osborne NJ (2013) Associations between socioeconomic status and environmental toxicant concentrations in adults in the USA: NHANES 2001-2010. Environ Int 59:328-335

20. Seo J-W, Kim B-G, Kim Y-M, Kim R-B, Chung J-Y, Lee K-M, Hong Y-S (2015) Trend of blood lead, mercury, and cadmium levels in Korean population: data analysis of the Korea National Health and Nutrition Examination Survey. Environ Monit Assess 187:146

21. Barrera G, Bunout D, Gattás V, de la Maza MP, Leiva L, Hirsch S (2004) A high body mass index protects against femoral neck osteoporosis in healthy elderly subjects. Nutrition 20:769-771

22. Jung SY, Kim S, Lee K, Kim JY, Bae WK, Lee K, Han J-S, Kim S (2015) Association between secondhand smoke exposure and blood lead and cadmium concentration in community dwelling women: the fifth Korea National Health and Nutrition Examination Survey (2010-2012). BMJ Open 5:e008218

23. Jung-Choi K-H, Khang Y-H, Cho H-J (2012) Hidden female smokers in Asia: a comparison of self-reported with cotinineverified smoking prevalence rates in representative national data from an Asian population. Tob Control 21:536-542

24. Haufroid V, Lison D (1998) Urinary cotinine as a tobacco-smoke exposure index: a minireview. Int Arch Occup Environ Health 71: $162-168$

25. Myong J-P, Kim H-R, Choi S, Koo J-W (2013) Dose-related effect of urinary cotinine levels on bone mineral density among Korean females. Osteoporos Int 24:1339-1346

26. Jang H-D, Hong J-Y, Han K, Lee JC, Shin B-J, Choi S-W, Suh SW, Yang J-H, Park S-Y, Bang C (2017) Relationship between bone mineral density and alcohol intake: a nationwide health survey analysis of postmenopausal women. PLoS One 12:e0180132

27. Holick MF (2004) Vitamin D: importance in the prevention of cancers, type 1 diabetes, heart disease, and osteoporosis. Am J Clin Nutr 79:362-371

28. Lee H-S, Cho Y-H, Park S-O, Kye S-H, Kim B-H, Hahm T-S, Kim M, Lee JO, C-i K (2006) Dietary exposure of the Korean population to arsenic, cadmium, lead and mercury. J Food Compos Anal 19: $\mathrm{S} 31-\mathrm{S} 37$

29. Warensjö E, Byberg L, Melhus H, Gedeborg R, Mallmin H, Wolk A, Michaëlsson K (2011) Dietary calcium intake and risk of fracture and osteoporosis: prospective longitudinal cohort study. Bmj $342: \mathrm{d} 1473$ 
30. Kim N-S, Lee B-K (2010) Blood total mercury and fish consumption in the Korean general population in KNHANES III, 2005. Sci Total Environ 408:4841-4847

31. Bagger YZ, Tankó LB, Alexandersen P, Hansen HB, Møllgaard A, Ravn P, Qvist P, Kanis JA, Christiansen C (2004) Two to three years of hormone replacement treatment in healthy women have long-term preventive effects on bone mass and osteoporotic fractures: the PERF study. Bone 34:728-735

32. Liu S, Lebrun C (2006) Effect of oral contraceptives and hormone replacement therapy on bone mineral density in premenopausal and perimenopausal women: a systematic review. Br J Sports Med 40: $11-24$

33. Lee K-S, Bae S-H, Lee SH, Lee J, Lee DR (2014) New reference data on bone mineral density and the prevalence of osteoporosis in Korean adults aged 50 years or older: the Korea National Health and Nutrition Examination Survey 2008-2010. J Korean Med Sci 29:1514-1522

34. Borné Y, Fagerberg B, Persson M, Östling G, Söderholm M, Hedblad B, Sallsten G, Barregard L, Engström G (2017) Cadmium, carotid atherosclerosis, and incidence of ischemic stroke. J Am Heart Assoc 6:e006415

35. Poole KE, Reeve J, Warburton EA (2002) Falls, fractures, and osteoporosis after stroke: time to think about protection? Stroke 33:1432-1436

36. Drake MT (2013) Osteoporosis and cancer. Curr Osteoporos Rep 11:163-170

37. Alfvén T, Järup L, Elinder CG (2002) Cadmium and lead in blood in relation to low bone mineral density and tubular proteinuria. Environ Health Perspect 110:699-702

38. Chen X, Gan C, Zhu G, Jin T (2013) Benchmark dose for estimation of cadmium reference level for osteoporosis in a Chinese female population. Food Chem Toxicol 55:592-595
39. Youness ER, Mohammed NA, Morsy FA (2012) Cadmium impact and osteoporosis: mechanism of action. Toxicol Mech Methods 22: $560-567$

40. Imai K, Keele L, Tingley D (2010) A general approach to causal mediation analysis. Psychol Methods 15:309-334

41. Huh JH, Choi SI, Lim JS, Chung CH, Shin JY, Lee MY (2015) Lower serum creatinine is associated with low bone mineral density in subjects without overt nephropathy. PLoS One 10:e133062

42. Tellez-Plaza M, Navas-Acien A, Crainiceanu CM, Guallar E (2008) Cadmium exposure and hypertension in the 1999-2004 National Health and Nutrition Examination Survey (NHANES). Environ Health Perspect 116:51-56

43. Adams SV, Newcomb PA (2014) Cadmium blood and urine concentrations as measures of exposure: NHANES 1999-2010. J Expo Sci Environ Epidemiol 24:163-170

44. Shimbo S, Zhang ZW, Moon CS, Watanabe T, Nakatsuka H, Matsuda-Inoguchi N, Higashikawa K, Ikeda M (2000) Correlation between urine and blood concentrations, and dietary intake of cadmium and lead among women in the general population of Japan. Int Arch Occup Environ Health 73:163-170

45. Burm E, Ha M, Kwon H-J (2015) Association between blood cadmium level and bone mineral density reduction modified by renal function in young and middle-aged men. J Trace Elem Med Biol 32:60-65

46. Järup L, Persson B, Elinder CG (1997) Blood cadmium as an indicator of dose in a long-term follow-up of workers previously exposed to cadmium. Scand J Work Environ Health 23:31-36

Publisher's note Springer Nature remains neutral with regard to jurisdictional claims in published maps and institutional affiliations. 